\title{
CARACTERIZACIÓN FISICOQUÍMICA DE LA MADURACIÓN DEL PLÁTANO DOMINICO-HARTÓN (Musa $A A B$ Simmonds)
}

\author{
PHYSICOCHEMICAL CHARACTERIZATION OF RIPENING OF THE DOMINICO-HARTON PLANTAIN FRUIT \\ (Musa AAB Simmonds)
}

\author{
Deiby Beltrán Giraldo, John Alexander Velásquez Téllez, Germán Antonio Giraldo Giraldo \\ Universidad del Quindío, facultad de ciencias básicas y tecnologías, programa de química, laboratorio de diseño de nuevos productos, \\ grupo de investigación: agroindustria de frutas tropicales.
}

Fecha de recibido: Febrero 3 de 2010

Fecha de aceptado: Junio 9 de 2010

Correspondencia: programa de química, laboratorio de diseño de nuevos productos, Universidad del Quindío, Av. Bolívar calle 12 norte Armenia Quindío. Correo electrónico: deibybg@hotmail.com

\section{RESUMEN}

El plátano Dominico Hartón (Musa AAB Simmonds) es de alto impacto económico en el departamento del Quindío, debido a la falta de estudios se realizó la caracterización fisicoquímica del plátano durante la etapa de maduración, el estudio se llevó a cabo con frutos en estado hecho, cosechando a las dieciocho semanas después de floración, se realizó la evaluación poscosecha durante catorce días, haciendo análisis por triplicado de los cambios fisicoquímicos de peso, color, índice de transpiración, índice de respiración, firmeza, $\mathrm{pH}$, acidez, actividad de agua y grados brix. Los resultados demostraron variación de peso cercanas al $25 \%$, en color los rangos para $L^{*}$ fueron entre 53.53 a 63.93, para $a^{*}$ entre -13.19 a 7.91 y para $b^{*}$ entre 34.04 a 49.20 , la firmeza paso de 22.78 a $3.41 \mathrm{~N} / \mathrm{seg}$., el pH de 5.8 a 4.1, la actividad de agua de 0.997 a 0.962 y los grados brix de 3.0 a 22.2 , los parámetros de maduración evidenciaron comportamiento climatérico. Durante la maduración se evidencia perdida de peso, cambio de color, disminución en la firmeza, el pHy la actividad de agua, y un aumento en los grados brix.

Palabras clave adicionales: Plátano Dominico-Hartón, poscosecha, maduración, índice de respiración y transpiración.

\section{ABSTRACT}

The banana Dominico Hartón (Musa AAB Simmonds) is of high economic impact in the department of Quindio. Due to lack of studies we carried out the characterization of the plantains during the stage of maturity, harvested to 18 weeks after flowering, the assessment postharvest was after 14 days, doing analysis in triplicate of physicochemical changes of weight, color, index of sweating, index of respiration, firmness, $\mathrm{pH}$, heartburn, water activity and Brix degrees. The results showed weight loses approximately $25 \%$, range of color had variation for example L* from 53.53 to 63.93 , for a* from -13.19 to 7.91 and for b* from 34.04 to 49.20 , the firmness decreased from 22.78 to $3.41 \mathrm{~N} / \mathrm{seg}$, the pH decreased from 5.8 to 4.1 , water activity from 0.997 to 0.962 and brix degrees increased from 3.0 to 22.2 . The maturation parameters showed climateric characteristics. During maturation is evident the weight loss, color change, decrease in firmness, $\mathrm{pH}$ and water activity and an increase in the brix degrees.

Key words: Plantain Dominico-Hartón, Post-reaps, Maturation, Index of respiration and transpiration

\section{INTRODUCCIÓN}

En Colombia existen actualmente 350.000 hectáreas del cultivo de plátano, la mayor parte de esta se encuentra en la zona cafetera central, de las cuales 10.000 hectáreas son para exportación a los mercados de Estados Unidos, Europa y las Antillas. EL plátano se destina principalmente al consumo en fresco y solo una pequeña proporción (1\%) para la agroindustria nacional (1).

El manejo poscosecha debe tener en cuenta los cambios fisicoquímicos del fruto, los que se evidencian como cambios estructurales, bioquímicos y físicos específicos para cada fruto, lo cual tiene estrecha relación con las características y la calidad del mismo. Arcila et al (1998) reportan pérdidas de peso en plátanos dominico Hartón y FIHA 21 a medida que transcurre el periodo de maduración
(2), también manifiestan que al inicio del proceso maduración del plátano presentó $0.5 \%$ de acido málico (3), Salvador et al. (1998) realizaron ensayos de penetración y flexión en $M$. cavendish AAA y $M$. paradisiaca AAB, obteniendo las curvas respectivas para cada ensayo Bugaud, Daribo y Dubois (2007) hacen un estudio de color y textura en frutos de banano $M$. cavendish AAA y $M$. paradisiaca $\mathrm{AAB}$ almacenados a $20 \circ \mathrm{C}$ (5)

El objetivo de este trabajo fue evaluar los cambios fisicoquímicos en la etapa de maduración del plátano Dominico-Hartón (Musa AAB Simmonds), a partir de las variables de peso, color, índice de transpiración, índice de respiración, firmeza, $\mathrm{pH}$, actividad de agua y grados brix, con el propósito de establecer las variaciones durante la etapa poscosecha. 


\section{Materiales y métodos}

\section{Recolección de las muestras}

Las muestras de plátano Dominico-Hartón (Musa AAB Simmonds) se recolectaron a las 18 semanas de floración, en la finca la Holanda de la vereda murillo $\mathrm{Km} 8$ vía Tebaida ubicada en el departamento del Quindío, a 1450 msnm, en estado de maduración hecho (cosecha).

\section{Tratamiento de las muestras}

Los plátanos se clasificaron de acuerdo a su longitud y diámetro, además se eliminaron los frutos con daños mecánicos, microbiológicos o por insectos y se dejaron a temperatura ambiente para su maduración $\left(23^{\circ} \mathrm{C}\right)$.

\section{Caracterización}

La caracterización de los frutos se realizó evaluando parámetros de peso, color, índice de transpiración y respiración, firmeza, $\mathrm{pH}$, acidez, actividad de agua y grados brix, desde el momento de la cosecha hasta estado senescente, bajo condiciones de experimentación de $23^{\circ} \mathrm{C} y$ $74 \%$ de humedad relativa.

\section{Métodos de análisis}

Los análisis realizados a las muestras en los diferentes estados de maduración fueron; el índice de transpiración se midió con base a la pérdida de agua representada en la pérdida de peso del fruto de plátano, el índice de respiración se determinó por el método de pettenkofer, La textura se midió en un texturometro TA. XT plus, en modo de compresión, empleando la sonda metálica P/0.5S (1 12 “ $\varnothing$ spherical stainless), con un adaptador de prueba AD/100 y una plataforma HDP/90. Los parámetros empleados fueron: velocidad del ensayo $1.1 \mathrm{~mm} / \mathrm{seg}$, velocidad después del ensayo $10.0 \mathrm{~mm} / \mathrm{seg}$ y fuerza de $5.0 \mathrm{~g}$, el $\mathrm{pH}$ se determinó por medio de un potenciómetro IQ-240, introduciendo 1.0 $\mathrm{cm}$ el electrodo para sólidos en la pulpa de plátano. Los grados Brix se midieron en un refractómetro de mesa (Thermo Electron Corporation) manteniendo una temperatura constante de 20 ㅇ. La acidez de las muestras de pulpa se determinó mediante un análisis volumétrico con hidróxido de sodio $0.1 \mathrm{~N}$, el resultado se utilizó para calcular el ácido presente, como ácido málico. La actividad de agua se determinó utilizando un higrómetro de punto de roció Decagon Devices, Inc Aqualab Model series 3 TE. El Color se determinó por medio de un colorímetro Minolta CR-10 en el sistema CIE-LAB. Los valores triestimulo $L^{*}, a^{*}$ y $b^{*}$ fueron suministrados en forma directa por el equipo, para calcular el croma métrico ( $\left.\mathrm{C}^{*}\right)$ y tono angular ( $\mathrm{h}$ ) $)$, se utilizaron las ecuaciones 1 y 2 (6).

$$
\begin{array}{ll}
\mathrm{C}^{*}=\left(\mathrm{a}^{2}+\mathrm{b}^{* 2}\right)^{1 / 2} & \text { (ecuación } 1) \\
\mathrm{h} \mathrm{o}=\arctan \left(\mathrm{b}^{*} / \mathrm{a} *\right) & \text { (ecuación 2) }
\end{array}
$$

\section{Resultados y discusión}

La frutos de plátano en estado hecho, se clasificaron teniendo en cuenta longitudes entre 28 y $30 \mathrm{~cm}$, diámetros entre 48 a $58 \mathrm{~mm}$, parámetros de color fueron para $L^{*}$ entre 52 y 54; para a* entre -14 y-11 y para b* entre 32 y 35.

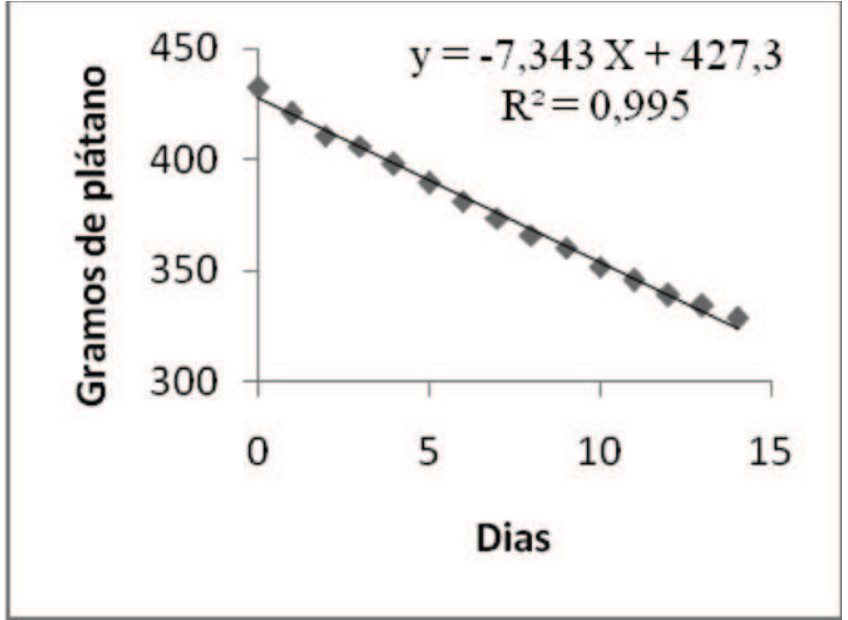

Figura 1. Pérdidas de peso durante la maduración de plátano Dominico-Hartón,

En la figura 1 se evidencia una pérdida constante de la masa a medida que avanza el proceso de maduración, con un coeficiente de relación de 0.995 lo cual muestra la relación lineal del tiempo en poscosecha con la pérdida de masa en los frutos, estos cambios de peso son atribuidos a la transpiración (pérdida de agua y compuestos volátiles), alcanzando el día 14 una disminución cercana al 25\% en peso en los últimos días se observó un notable deterioro en la epidermis o cáscara, y disminución de la textura (7).

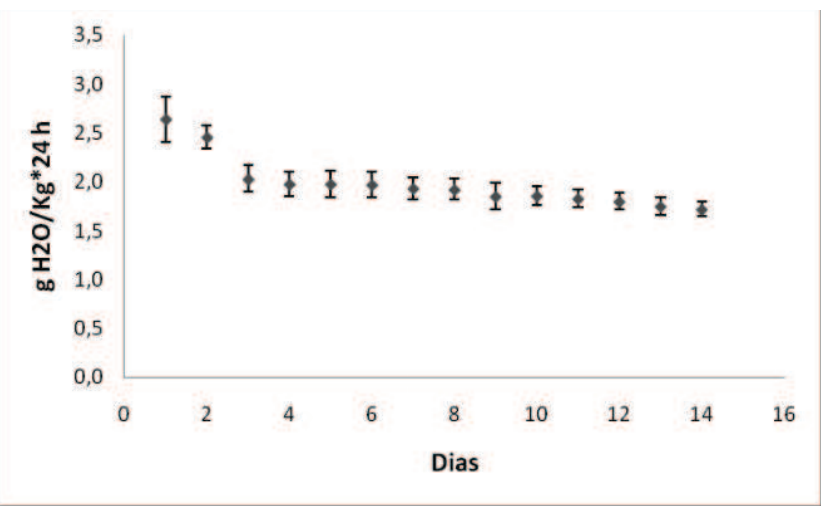

Figura 2. Índice de transpiración durante la maduración de plátano Dominico-Hartón.

El índice de transpiración se muestra en la figura 2, observándose que esta disminuye gradualmente durante el periodo de maduración del plátano, coincidiendo con lo mencionado por Arcila et al. (2), quienes reportan pérdida gradual de peso en plátanos dominico Hartón y FIHA 21 a medida que transcurre el periodo de maduración, esta pérdida de agua se traduce en una reducción de peso y pérdida de turgencia del producto.

El fenómeno de transpiración se inicia cuando las células presentan turgencia y los estomas se encuentran abiertos, lo que genera intercambio gaseoso, dando mayor pérdida de peso (8). 
El índice de respiración de la figura 3 corresponde a un comportamiento con características climatéricas; en él se identifican sus tres etapas: en la primera se desarrollaron algunas de sus características organolépticas con producción de $\mathrm{CO} 2$ creciente, alcanzando un máximo pico de maduración el día quinto.

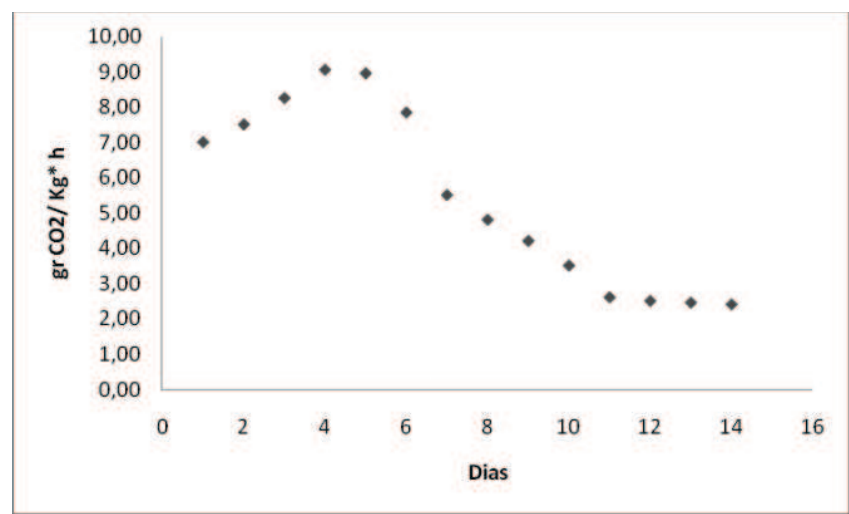

Figura 3. Índice de respiración del plátano Dominico-Hartón en poscosecha.

La segunda etapa, presenta la producción constante de $\mathrm{CO} 2$ alcanzando la madurez de consumo entre los días 5 y 6 , donde se logran las características fisicoquímicas y sensoriales ideales para el consumo. En la tercera etapa el comportamiento del índice de madurez corresponde a la senescencia o sobre maduración, donde los atributos de consumo empiezan a afectarse por deterioro de la cáscara y degradación del fruto $(9,10)$. Posiblemente la temperatura fue el factor que ejerció mayor influencia sobre la velocidad de respiración del plátano $(11,12,13)$.

Las frutas a medida que maduran se tornan más susceptibles de sufrir daños mecánicos durante el manejo poscosecha debido a cambios en la textura. En la figura 4 se muestra el cambio de firmeza del plátano durante la maduración, la disminución en firmeza se correspondió a la hidrólisis de almidones y pectinas, por el proceso degradativo de la lamina media y las paredes celulares, produciendo sacarosa y ácido galacturonico, generando una mayor flexibilidad en el material $(14,15,16)$.

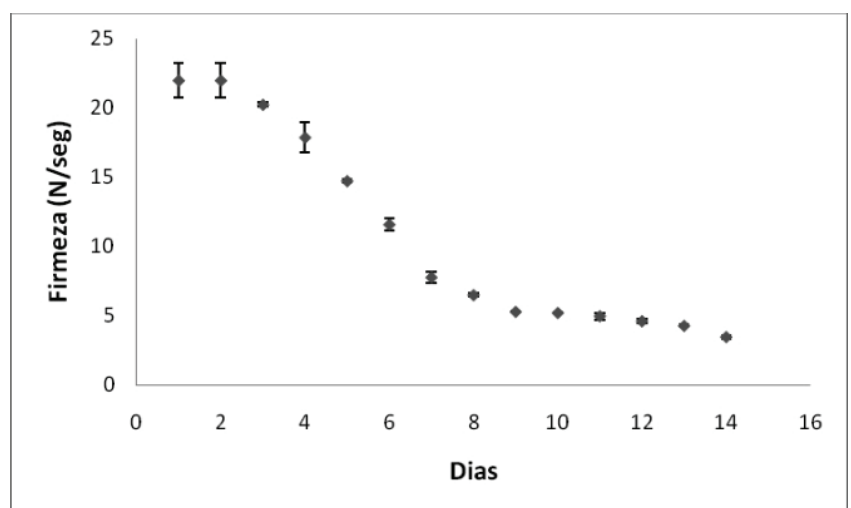

Figura 4. Firmeza durante la maduración de plátano Dominico-Hartón.
Al comparar los resultados con lo mencionado por Ciro y Osorio, Chávez y Franco, y Gómez et al. $(17,18,19)$ se confirmó que el grado de firmeza de una fruta cambia con el avance del proceso de maduración del fruto.

Salvador et al. (4) realizaron ensayos de penetración y flexión en $M$. cavendish AAA y $M$. paradisiaca $A A B$, obteniendo las curvas respectivas para cada ensayo. Al comparar los ensayos de penetración de plátano DominicoHartón (Musa AAB Simmonds) con el M. cavendish AAA, ambos ensayos presentaron una considerable disminución de la fuerza a medida que transcurrió el periodo de maduración, este comportamiento concuerda con lo observado en el índice de respiración y de transpiración del fruto, evidenciándose con el tiempo disminución de la resistencia del material vegetal (19).

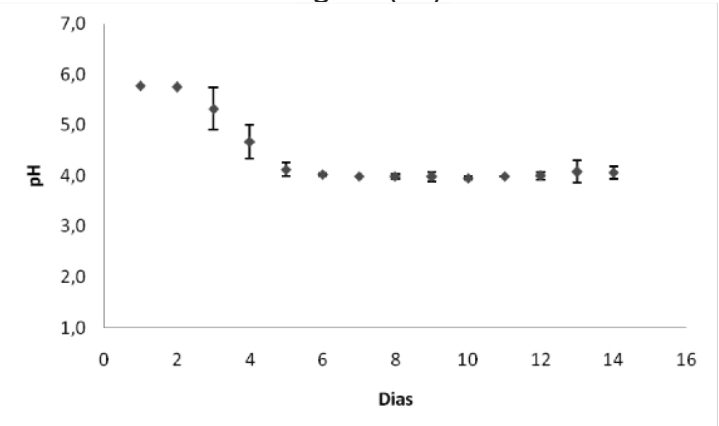

Figura 5. Cambio de pH durante la maduración de plátano Dominico-Hartón.

En el proceso de maduración, el pH disminuyó de 5,78 a 3,96 (figura 5), esto concuerda con lo reportado por Arcila et. al., (1998). En cuanto al comportamiento del pH, a partir del día sexto se observa un valor constante hasta el 11 día, a partir de este día hasta el 14 día, hay un leve aumento del $\mathrm{pH}$, que puede deberse al incremento de sacarosa y a la degradación de estructuras poliméricas del fruto.

Los azúcares son el componente mayoritario de los sólidos solubles totales (SST), por lo que éstos se usan como criterio para establecer normas de maduración de algunas frutas; además, su calidad comestible suele correlacionarse mejor con el contenido de SST.

El comportamiento de los grados Brix se muestra en la figura 6 , donde aumentan, conforme avanzan los días del periodo de maduración, confirmando lo reportado por Arcila et al. (1998) (3) afirmando que durante la maduración se presenta un incremento de los SST.

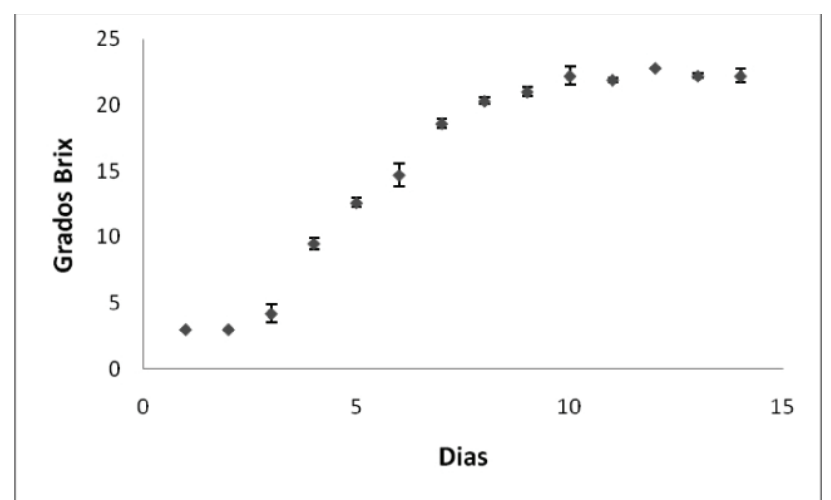

Figura 6. Grados Brix durante la maduración de plátano Dominico-Hartón.

Rev. Invest. Univ. Quindío (20): 166 - 170. Armenia - Colombia 
También se ha demostrado que los grados Brix aumentan durante la maduración debido a hidrólisis de almidones y disacáridos, además de la síntesis de monosacáridos (14, 20 ,21). El aumento progresivo de los grados Brix en la etapa de maduración y sobremaduración hacia el día doce, se debe a que el fruto presenta menor cantidad de agua (transpiración) y mayor concentración de azucares (respiración), originado por la mayor producción de etileno, quien da inicio al incremento del índice de respiración $(22,23)$. En la última etapa se mantiene un nivel constante de grados Brix, siendo la etapa de sobremaduracion, la cual se presenta a partir del día doce.

Tabla 1. Acidez (acido málico) durante la maduración de plátano Dominico-Hartón.

\begin{tabular}{cc}
\hline días & $\begin{array}{c}\text { \% Acido } \\
\text { málico/Kg } \\
\text { pulpa }\end{array}$ \\
\hline 1 & 0.4 \\
2 & 0.4 \\
3 & 0.5 \\
4 & 0.75 \\
5 & 0.9 \\
6 & 1.05 \\
7 & 1.1 \\
8 & 1.2 \\
9 & 1.2 \\
10 & 1.2 \\
11 & 1.2 \\
12 & 1.25 \\
13 & 1.25 \\
14 & 1.2 \\
\hline
\end{tabular}

La generación de ácido málico interviene en el pH del plátano dominico hartón durante su maduración. Este se incrementó atreves de dicho proceso, lo cual coincide con lo reportado por Arcila et al (1998) afirmando que al inicio del proceso maduración presentó $0.5 \%$ de acido málico.

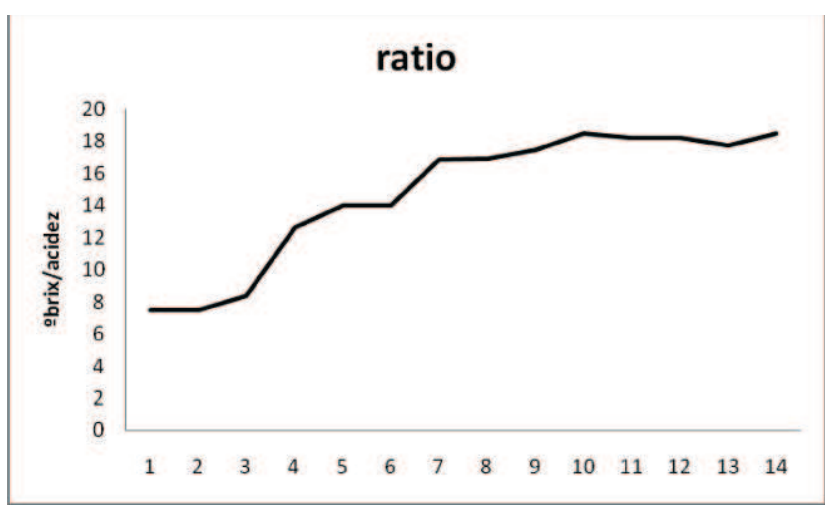

Figura 7. Ratio grados brix/acidez durante la maduración de plátano Dominico-Hartón
En la grafica 7 se observa una estrecha relación entre los grados brix y la acidez, lo que presenta un aumento representativo de la madurez del fruto siendo significativo en el día 7, como el fin de la maduración.

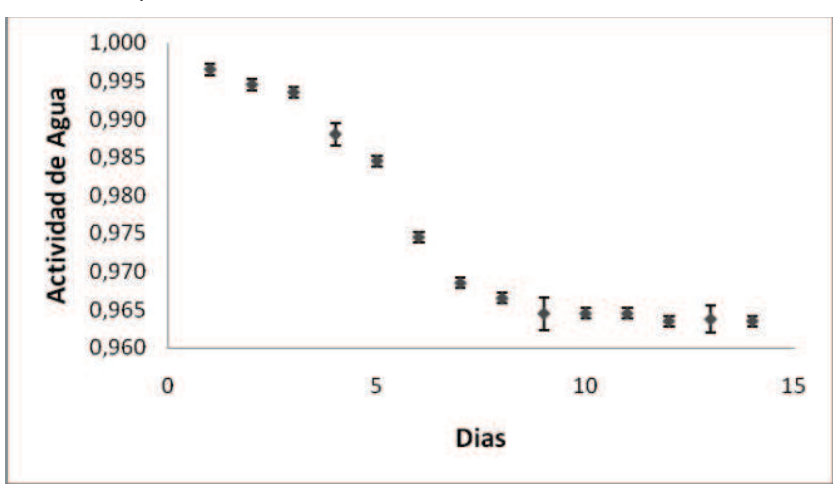

Figura 8. Actividad de agua (aw) durante la maduración de plátano Dominico-Hartón.

Los niveles de actividad de agua durante la maduración se muestran en la figura 8, donde hay un decaimiento debido a que las aguas libres del fruto se convierten en aguas de reacción o ligadas presentes en las hidrólisis y transpiración. Estos resultados contrastan con los obtenidos por Arrieta et al. (24) quien afirma que la de actividad de agua aumenta conforme trascurren los días Poscosecha, concluyendo en el día 7, siendo el fin de la madurez de consumo.

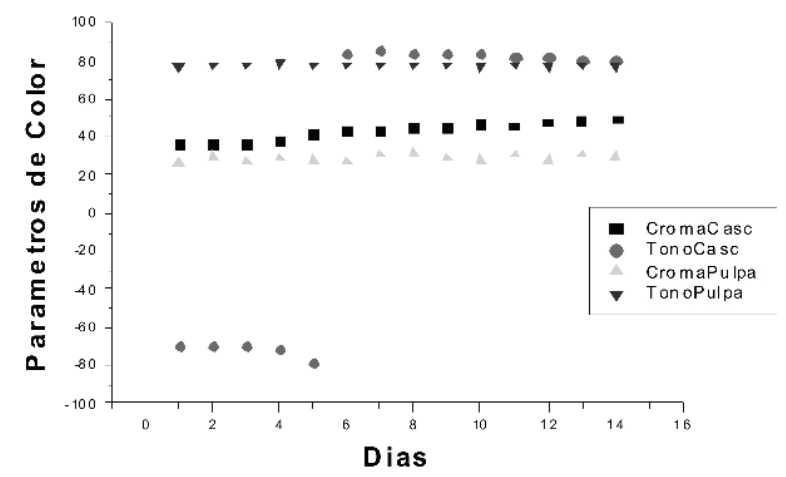

Figura 9. Parámetros de color durante la maduración de plátano Dominico-Hartón.

En la figura 9 se representan los parámetros de color en la pulpa y cascara del plátano, el color en la cáscara cambia de verde a amarillo durante la etapa de maduración, estos cambios se deben a la disminución del contenido de clorofila y al incremento en la síntesis de pigmentos de color amarillo, (carotenoides y antocianinas) (25). Resultados similares se encontraron en frutos de banano $M$. cavendish AAA y $M$. paradisiaca $A A B$ almacenados a 20 ㅇ C (5).

El análisis de la cromaticidad en la pulpa, no presentó diferencias apreciables desde el inicio de la poscosecha hasta la sobre-maduración, a pesar de encontrarse variabilidad en la lectura, lo que indica que los cambios de color que se observan en el desarrollo de la maduración en la pulpa, no están influenciados por los pigmentos responsables de la aparición de tonos fuertes. Las 
variaciones del color en la pulpa y la cáscara en el proceso de maduración se asocian probablemente a diferencias en el contenido del carotenoides en la fruta $(5,26)$.

\section{Conclusiones}

Los frutos de plátano Dominico Hartón durante la maduración presentaron un comportamiento climatérico, una variación en la firmeza durante el almacenamiento, y una disminución del pH debido a la aparición de acido málico, además de un incremento en concentración de grados Brix en el día doce debido al incremento de sólidos solubles. Estos parámetros permiten establecer cambios en las variables de las características del plátano DominicoHartón durante la postcosecha.

\section{BIBLIOGRAFÍA}

1. Ministerio de Agricultura y Desarrollo Rural. "La cadena de plátano en Colombia”. Observatorio Agrocadenas Colombia. Documento de trabajo, (2006). (102), 2.

2. Arcila M I, Giraldo G, Belalcázar S, Cayòn G. y Méndez J C. “Comportamiento poscosecha de los plátanos dominico Hartón y FHIA 21 en diferentes presentaciones". Memorias del seminario internacional sobre la producción de plátano. Armenia, Quindío, Colombia, del 4 al 8 de mayo de 1998, 256-260.

3. Arcila M I. "Aspectos sobre fisiología y manejo poscosecha del plátano en la región cafetera central". Memorias del seminario internacional sobre la producción de plátano. Armenia, Quindío, Colombia, (1998). 247-255.

4. Salvador A, Sanz T. Fiszman S M. "Changes in colour and texture and their relationship with eating quality during storage of two different dessert bananas". Postharvest Biology and Technology, (2007). 43, 319-325.

5. Bugaud C, Daribo M O, Dubois C. "Climatic conditions affect the texture and colour of Cavendish bananas". Grande Naine cultivar, (2007). 113, 238-243.

6. Gilbert E. "Medida de color". Espacios de color. 3a edición. (2001). 541.

7. Márquez C J, Otero C M, Cortés R M. "Cambios fisiológicos, texturales, fisicoquímicos y microestructurales del tomate de árbol (cyphomandra betacea s.) En poscosecha". Vitae 14(2).

8. Gardiazábal F, Rosenberg G. "El cultivo del chirimoyo. Valparaíso", Ediciones Universitarias de Valparaíso, (1993). P 152167.

9. Pórtela S. "Postharvest physiology and handling of tamarillos (Cyphomandra betacea)".Avances en Horticultura, (2001). 4(1), 40-50.

10. Villamizar F. "Manejo tecnológico poscosecha de frutas y hortalizas". Manual de prácticas. Bogotá: Universidad Nacional de Colombia, (2001.). 16-22.

11. Uinsma J, Paull R E. "Respiration during postharvest development of soursop fruit, Annona muricata L". Plant Phisiology, (1984). 76, 131-138.

12. Bhande M R, Ravindra T K, Goswami A. "Respiration rate of banana fruit under aerobic conditions at different storage temperatures". Journal of Food Engineering, (2008). 87, 116-123.

13. Kader A A. "Biochemical and physiological basis for effects of controlled and modified atmospheres on fruits and vegetables". Food Technology. (1986). vol 40, 99-104.

14. Fennema O. “Química de alimentos". Zaragoza. Editorial Zaragoza. (1993).

15. Park Y S, Jung S T, Gorinstein S. "Ethylene treatment of 'Hayward' kiwifruits (Actinidia deliciosa) during ripening and its influence on ethylene biosintesis and antioxidant activity". Scientia horticolturae, (2006). (108), 22-28.

16. Ross CW. Salisbury F B. "Fisiología de las plantas. Madrid", (2000). 276-278.

17. Ciro H J. Osorio J A. "Avance experimental de la ingeniería depostcosecha de frutas colombianas: resistencia mecánica para frutos de uchuva (physalis peruvianal)". Dyna, (2008). 75(154).

18. Hernández A, Wang J. García P A. "Impulse response of pear fruit and its relation to Magness-Taylor firmness during storage". Postharvest Biology and Technology, (2005). 35, 209-215.

19. Chávez S. Franco H. "Propiedades biomecánicas de los frutos, caso zarzamora”. En: Memoria IX Curso de Actualización, Frutales con Futuro en el Comercio Internacional. Fundación Salvador Sánchez. Colin Cictamex, (2000). 34, $329-335$.

20. Budde C. Polenta G, Lucangeli C, Murria R. "Air and immersion heat treatments affect ethylene production and organoleptic quality of "Dixiland' peaches". Postharvest Biology and Technology. (2006). (41), 32-37.

21. Gallo F. "Manual de fisiología, patología poscosecha y control de calidad en frutas y hortalizas". (1997). Armenia, Colombia, SENA.

22. Kumar R, Hoda M. “Fixation of maturity standards of guava (Psidium guajava L.)”. Indian J. Hort, (1974). 31(2), $140-144$.

23. Laguado N, Marín M, Oliveros S. "Características físicoquímicas y fisiológicas de frutos de guayaba de los tipos Criolla Roja y San Miguel procedentes de dos plantaciones comerciales" Rev. Fac. Agron. (1997). 16, 382-397.

24. Arrieta A J. Ubaldo M. Barrera J L. "Caracterización físico-química del proceso de maduración del plátano 'Papocho' (Musa ABB Simmonds) (2006). 24(1), 48-53.

25. Burton W G. "Post-harvest physiology of food crops". Longman Group Ltd, London (1982). 339.

26. Salvador S, Fiszman M, Calvo C. "Influencia del tiempo y temperatura de almacenamiento en el color externo de plátanos". (2002). 1-3. 